\title{
Modelado de la logística de distribución tendiente a la mitigación del "efecto látigo", caso sector bebidas en Perú y Colombia
}

\author{
Distribution logistics modeling aimed at the "bullwhip effect" \\ mitigation, drinks industry case in Perú and Colombia
}

Felipe Néstor Gutarra Meza', Diego José Gómez Montoya²

Universidad Continental

\section{RESUMEN}

Objetivos: Generar un modelo matemático del sistema de distribución de bebidas, que permita mitigar el "efecto látigo" sobre la información de demanda, tomando información histórica de dos empresas de bebidas ubicadas en Perú y Colombia. Métodos: Se utilizó la dinámica de sistemas, modelos de Forrester y técnicas de predicción de demanda. Los procedimientos del estudio fueron: se hizo un reconocimiento de los antecedentes de estudios referentes a la logística de distribución; se identificaron las variables que tienen incidencia en el sistema de distribución de empresas de bebidas; se determinaron las relaciones causales existentes entre las variables identificadas, estableciéndose el modelo que describe el sistema de distribución de bebidas, integrando los actores y eslabones intervinientes, validándose el modelo propuesto con información histórica de empresas del sector bebidas; finalmente, se plantearon recomendaciones o medidas de control tendientes a la mitigación del "efecto látigo". Resultados: Se evidencia que la fluctuación alrededor de un inventario efectivo de 0 , muestra un patrón cíclico a partir del mes 19, con demoras semanales (y a partir del mes 34 , con demoras mensuales). El tipo de demanda (constante o estocástica), no implica diferencias significativas en el comportamiento del inventario efectivo, solo un retraso más. Conclusiones: Se determinaron las variables necesarias para modelar el sistema; también se lograron determinar las relaciones causales existentes entre las variables identificadas, mediante la elaboración de un diagrama causal

que muestra la dirección y el sentido de cada relación de incidencia (positiva o negativa).

Palabras clave: Sector bebidas, cadena de abastecimiento, modelo matemático, "efecto látigo", demanda estocástica, diagrama de Forrester.

1 Magíster en Docencia e Investigación en Educación Superior. Coordinador de la E.A.P de Ingeniería Industrial de la Universidad Continental, Perú.

2 Magíster en Ingeniería Industrial. Investigador de la Universidad Manuela Beltrán, Colombia. 


\section{ABSTRACT}

Objectives: To generate a mathematical model from the drinks distribution system, which allows to mitigate the "bullwhip effect" on the demand information, taking historical information from two drinks' companies located in Peru and Colombia. Methods: We used system dynamics, Forrester models and demand forecasting's techniques. The procedures of the study were: it was realized a background recognition about studies related to the distribution logistics; it was identified the variables which have incidence in the drinks companies' distribution system; it was determined the existent causal relationships were determined between the identified variables. Establishing the model which describes the drinks' distribution system, integrating involved actors and steps, validating the proposed model with historical information from drinks' companies. Finally, recommendations or control measures were raised aimed at the "bullwhip effect" mitigation. Results: There is evidence that the fluctuation around an effective inventory of 0 , shows a cyclical pattern from month 19, with weekly delays (and from month 34, with monthly delays). The demand type (constant or stochastic) involves no significant differences in the effective inventory behavior, only one more delay. Conclusions: Were determined the necessary variables to model the system; also it was able to determine the existent causal relationships between the identified variables, through the causal diagram elaboration, it shows the direction and sense of each incidence's relationship (positive or negative).

Keywords: Drinks' sector, supply chain, mathematical model, "bullwhip effect", stochastic demand, Forrester diagram.

\section{INTRODUCCIÓN}

El estudio pretende generar un modelo matemático del sistema de distribución de bebidas, que permita mitigar el "efecto látigo" sobre la información de demanda. Para ello postulamos metas parciales como: reconocer antecedentes, identificar variables incidentes en el sistema de distribución de bebidas, determinar las relaciones causales entre dichas variables, establecer un modelo que describa el sistema e incluya los actores y eslabones intervinientes, utilizar el modelo propuesto con información histórica de empresas del sector bebidas y plantear recomendaciones - medidas de control tendientes a la mitigación del fenómeno.

La investigación tiene un elevado componente cuantitativo, sin embargo se opta por trabajar con una hipótesis cualitativa que plantea que es posible modelar la cadena de suministro, de tal forma que puedan determinarse algunas medidas para mitigar las consecuencias del "efecto látigo" sobre un sistema logístico.

El fenómeno estudiado, el "efecto látigo" o "bullwhip", consiste en la distorsión entre la información de demanda que emite el mercado y la que capta el fabricante y es trascendental su comprensión y regulación, ya que trae como consecuencias el incremento en los costos de mantener inventario, incertidumbre durante la planeación de la producción e incumplimiento de la demanda que a su vez impacta sobre la imagen y el posicionamiento de marcas y empresas.

La investigación relacionada con el problema de la amplificación de los pedidos en los sistemas de producción y distribución de forma documentada se remonta a inicios del siglo anterior (1).

Algunas causas comunes que provocan el fenómeno son las perturbaciones debidas a sesgos introducidos en la demanda, las perturbaciones introducidas por los tiempos de repuesta, las prácticas logísticas inapropiadas y la alteración de las condiciones de la red de distribución (2).

Sancar realiza experimentos asumiendo parámetros para inducir la estacionalidad 
y variación de la demanda; finalmente concluye que la eliminación del "efecto látigo" es posible si se toman las acciones correctas en los negocios, tales como incrementar las habilidades de comunicación, cortar tiempos de entrega o implementar un sistema de información (3).

Los estudios anteriores son un esfuerzo por hacer más eficiente la cadena de suministro, entendiendo esta como una red de organizaciones interrelacionadas que intervienen en diferentes fases del proceso productivo mediante actividades que pretenden añadir valor, desde el punto de vista del cliente, al producto, bien o servicio (4).

Según Stadtler, una cadena de suministro consta de dos o más organizaciones legalmente separadas que están conectadas por flujos de materiales, de información y financieros. Un problema que sufren los miembros de dichas redes logísticas es el temido "efecto látigo", fenómeno que se refiere al aumento de la variabilidad en los pedidos incluso cuando la demanda del mercado es estable (5).

La conceptualización de cadena de suministro ya aparece en los trabajos de Forrester, cuando sugirió que el éxito de las empresas dependía de la interacción entre los flujos de información, materiales, pedidos, dinero, mano de obra y equipos; también cuando planteó que la comprensión y control de estos flujos es el trabajo principal de la gestión (6). Forrester utilizó en sus experimentos de simulación una cadena de suministro compuesto por cuatro niveles: la fábrica, el distribuidor, el mayorista y el minorista (Figura 1).

El "efecto látigo" determina una continua alteración de los planes de producción y una frecuente inestabilidad de los inventarios; lo anterior provoca un aumento de costes innecesarios para las organizaciones en el proceso de creación de valor. Se ha estimado que las consecuencias económicas de este síntoma frecuente y dañino del sistema logístico pueden incidir en un incremento de costes innecesarios de hasta el $30 \%$ de las ganancias de la red (7).

\section{MATERIAL Y MÉTODOS}

Esta investigación tiene un componente explicativo, por encontrar el sentido de la incidencia o de las relaciones entre variables del sistema; y otro de tipo correlacional, al determinar la magnitud de las incidencias - la forma en la que se presentan las relaciones, a modo de ecuaciones.

El enfoque es principalmente cuantitativo. Desde otra tipología de investigación, se puede asumir como una investigación aplicada (en dos grandes empresas de bebidas, tanto de Perú como de Colombia).

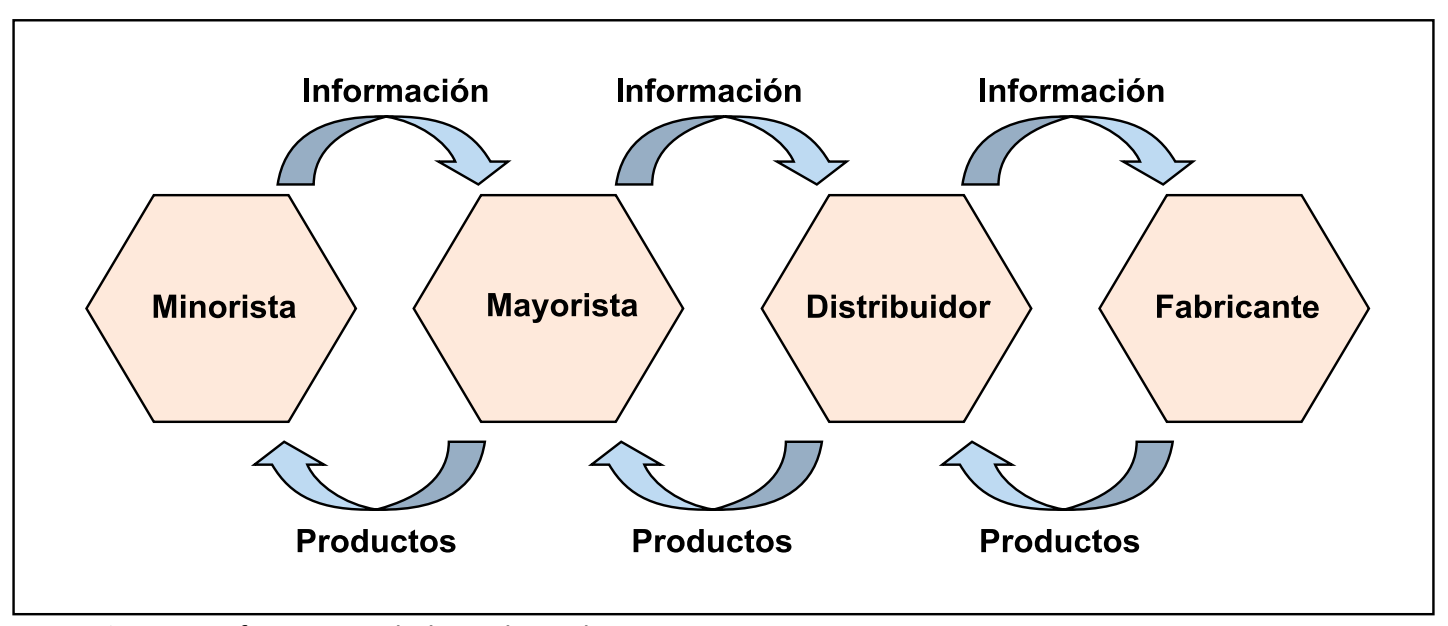

Figura $\mathrm{N}^{\circ} 1$ : Configuración de la cadena de suministro. 
Tabla $\mathrm{N}^{\circ}$ 1: Formato para levantamiento de datos en el eslabón de distribuidores.

\begin{tabular}{|c|c|c|c|c|c|c|}
\hline Período & $\begin{array}{l}\text { Pedido recibido de } \\
\text { los expendios }\end{array}$ & $\begin{array}{l}\text { Salida de } \\
\text { productos }\end{array}$ & $\begin{array}{l}\text { Entrada de } \\
\text { productos }\end{array}$ & $\begin{array}{l}\text { Inventario } \\
\text { final }\end{array}$ & $\begin{array}{l}\text { Demora en recibir la } \\
\text { información de } \\
\text { pedido }\end{array}$ & $\begin{array}{l}\text { Demora entre la } \\
\text { salida de producto } \\
\text { de distribuidores y la } \\
\text { entrada al inventario } \\
\text { de los expendios }\end{array}$ \\
\hline \multicolumn{7}{|l|}{1} \\
\hline \multicolumn{7}{|l|}{2} \\
\hline \multicolumn{7}{|l|}{3} \\
\hline \multicolumn{7}{|l|}{4} \\
\hline \multicolumn{7}{|l|}{5} \\
\hline \multicolumn{7}{|l|}{6} \\
\hline \multicolumn{7}{|l|}{7} \\
\hline \multicolumn{7}{|l|}{8} \\
\hline \multicolumn{7}{|l|}{9} \\
\hline 10 & & & & & & \\
\hline
\end{tabular}

Los métodos utilizados fueron la dinámica de sistemas, los modelos de Forrester y las técnicas de predicción de demanda.

En pensamiento sistémico, un sistema es una red de componentes, compleja, altamente interrelacionada que exhiben propiedades sinérgicas -el todo es mayor a la suma de sus partes- (8).

Básicamente son tres los principios o pilares fundamentales sobre los que se apoya la dinámica de sistemas: a) la existencia de bucles de realimentación, b) la existencia de no linealidad en las relaciones entre variables, y c) la existencia de retrasos en las relaciones entre variables (9).

El modelo desarrollado se genera con base en los trabajos realizados por Jay Forrester y otros profesionales del MIT (Massachusetts Institute of Technology) quienes estudiaron el fenómeno mediante el Juego de la Cerveza (10).

Las unidades de análisis fueron las empresas Ajeper (Perú) y Postobón (Colombia), así como los eslabones presentados en la cadena de suministro de algunas de sus bebidas. Se utilizarán como mínimo 10

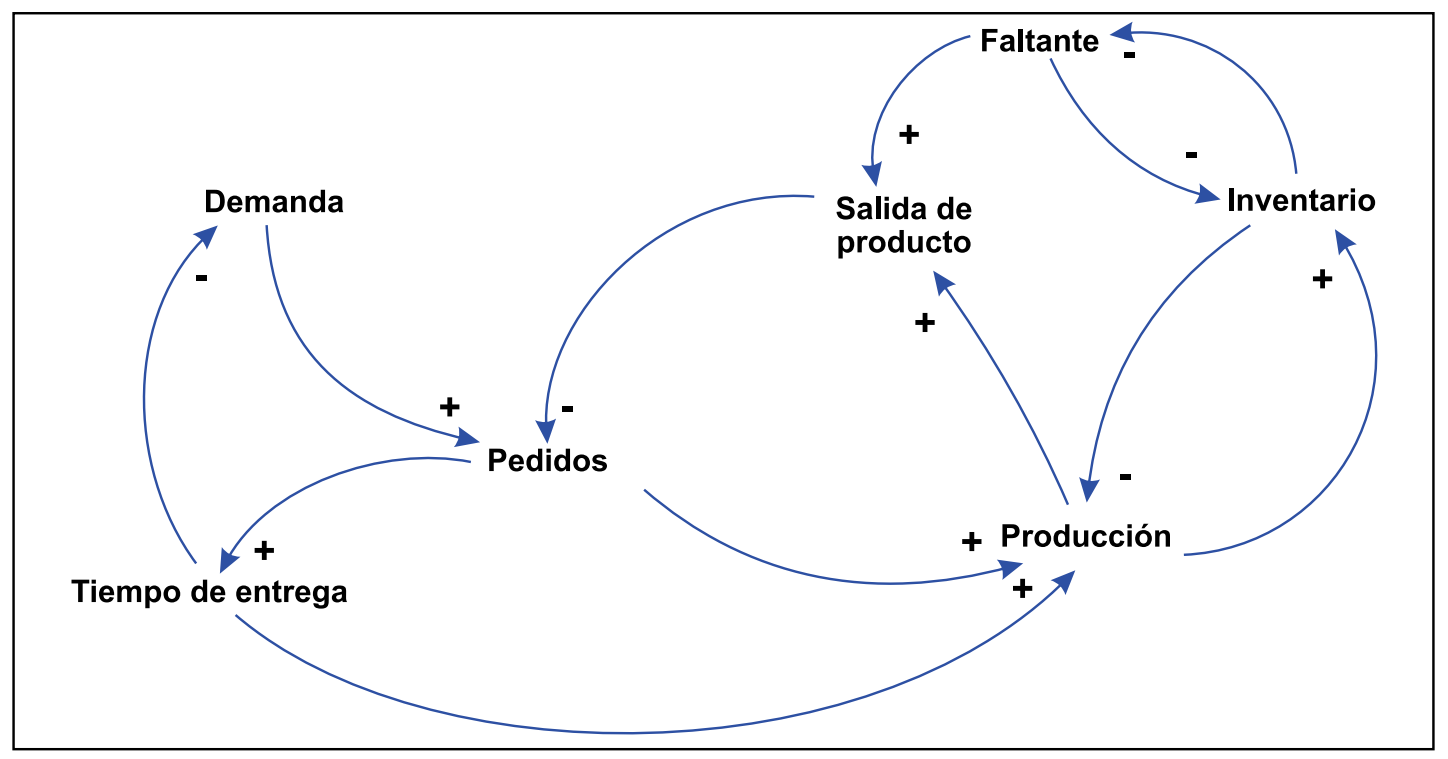

Figura $\mathrm{N}^{\circ}$ 2: Diagrama causal genérico. 
períodos históricos para la construcción de los modelos.

Los datos fueron obtenidos a partir de fuentes de información secundaria que poseen las empresas objeto de análisis y directamente de las áreas encargadas del manejo de cifras históricas. Con tal propósito, se diseñaron formatos para cada uno de los eslabones (expendios, distribuidores y fábrica). Presentamos el formato de distribuidores en la Tabla 1.

\section{RESULTADOS}

Se determinaron las variables necesarias para modelar el sistema, estas son: la cantidad demandada de producto por parte de los clientes o del mercado en cada período, las órdenes o los pedidos (cada período) que realiza cada eslabón al eslabón que lo provee, el tiempo en que llega la información de pedido entre un eslabón y otro, la cantidad de producto que se despacha cada período en cada eslabón, la cantidad de producto que ingresa -o que se produce, en el caso de la fábrica-, cada período en cada eslabón, los niveles de inventario y de faltante de producto en cada período para cada eslabón y los tiempos de entrega de producto entre eslabones. La interacción entre estas variables, se analiza mediante un diagrama causal (Figura 2).

El estudio de estas interacciones proporciona la base para la elaboración del diagrama de Forrester que integra a los tres eslabones utilizados (expendios, distribuidores y fábrica) y se presenta en la Figura 3.

La comprensión del diagrama inicia con la demanda que emiten los clientes; esta se

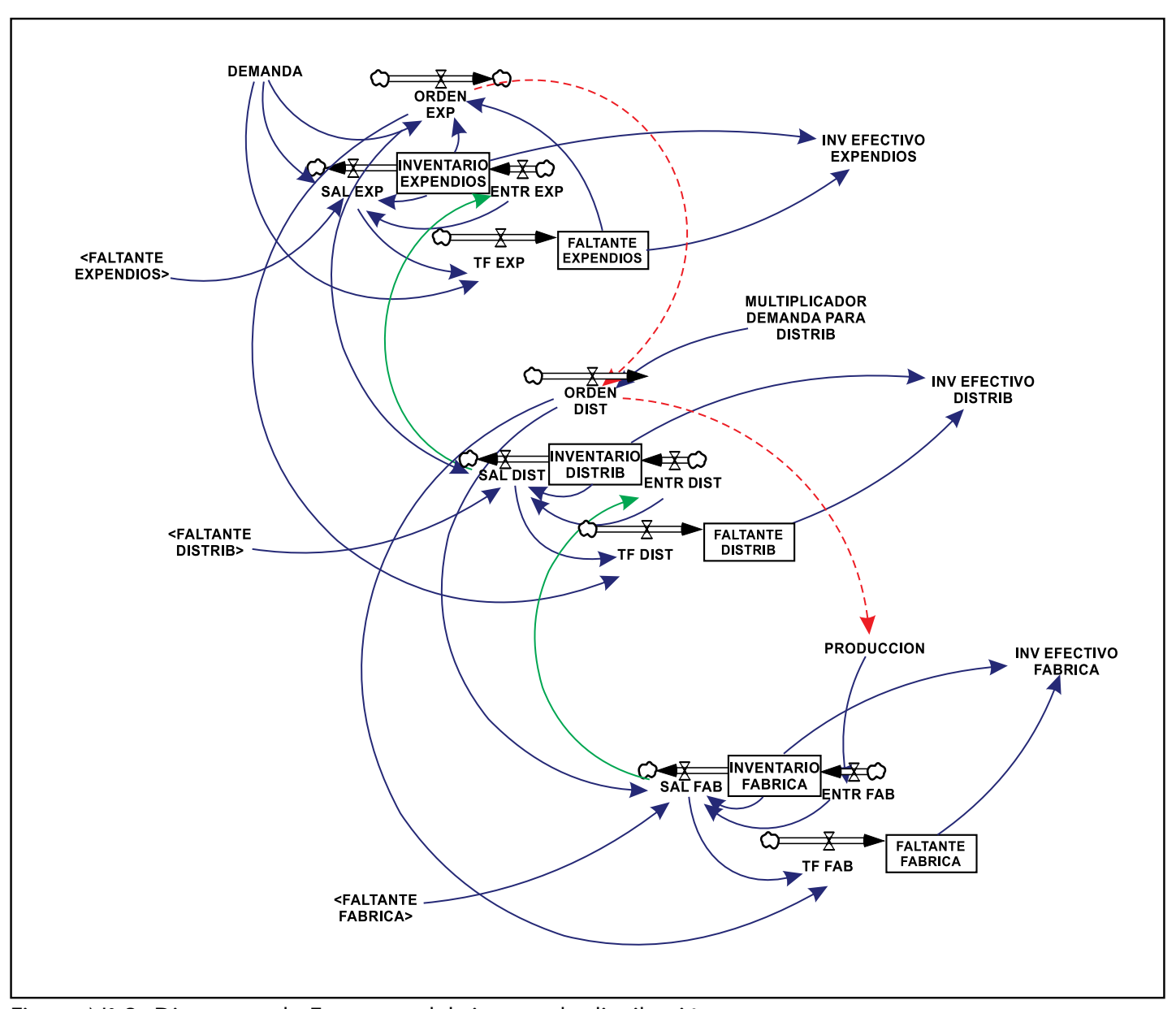

Figura $N^{\circ}$ 3: Diagrama de Forrester del sistema de distribución. 


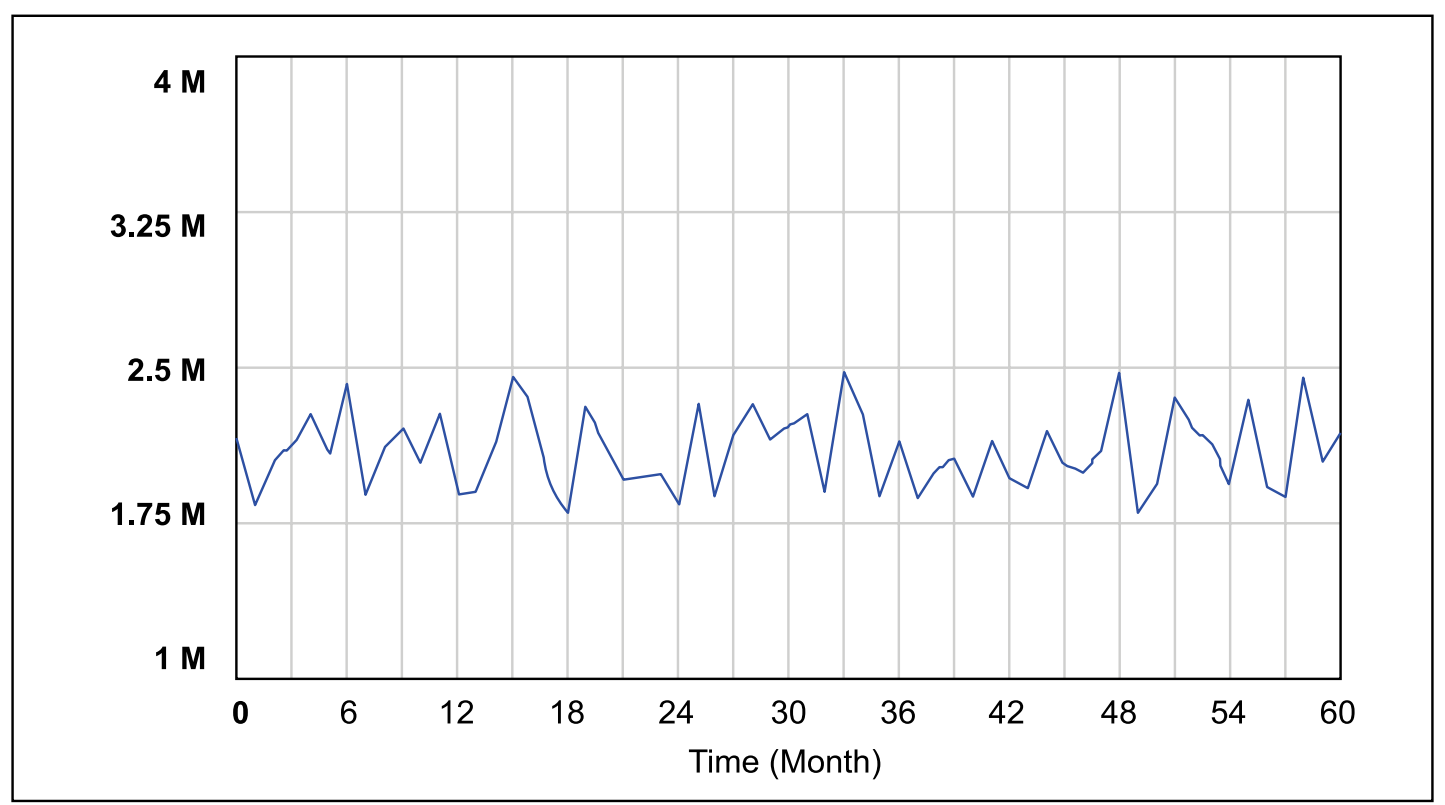

Figura $\mathrm{N}^{\circ}$ 4: Demanda simulada para Ajeper (durante 5 años)

representa mediante una variable auxiliar, que luego de ser analizada en el software estadístico StatFit, se determinó como una distribución estadística uniforme -tanto en Ajeper como en Bavaria -, con parámetros 1786550 y 2483680 (en el caso de Ajeper). En la Figura 4 se aprecia como oscilaría la demanda para Apejer en un horizonte de 60 meses (5 años).

La suma entre el inventario expendios y el faltante (teniendo en cuenta que este último se concibe como un inventario negativo), será el inventario efectivo; en las figuras 5, 6 y 7 se aprecia cómo se comporta en cada uno de los eslabones (expendios, distribuidores y fábrica, respectivamente). Cada uno de los colores indica un escenario (rojo: demoras en semanas, verde: demoras en meses, azul: demoras en semanas con demanda constante). Se evidencia que la fluctuación alrededor de un inventario efectivo de 0 ,

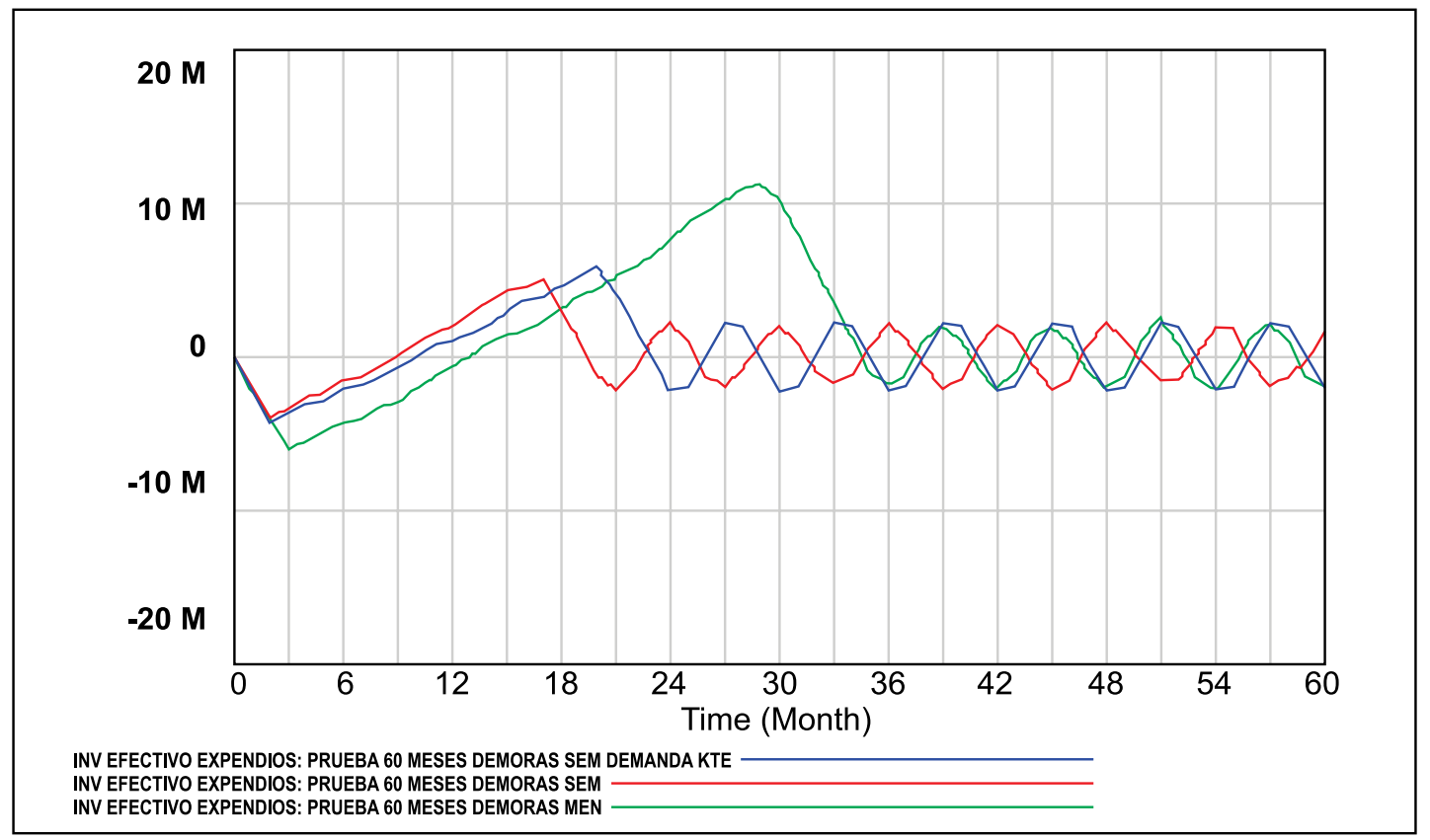

Figura $\mathrm{N}^{\circ}$ 5: Inventario efectivo de expendios. 


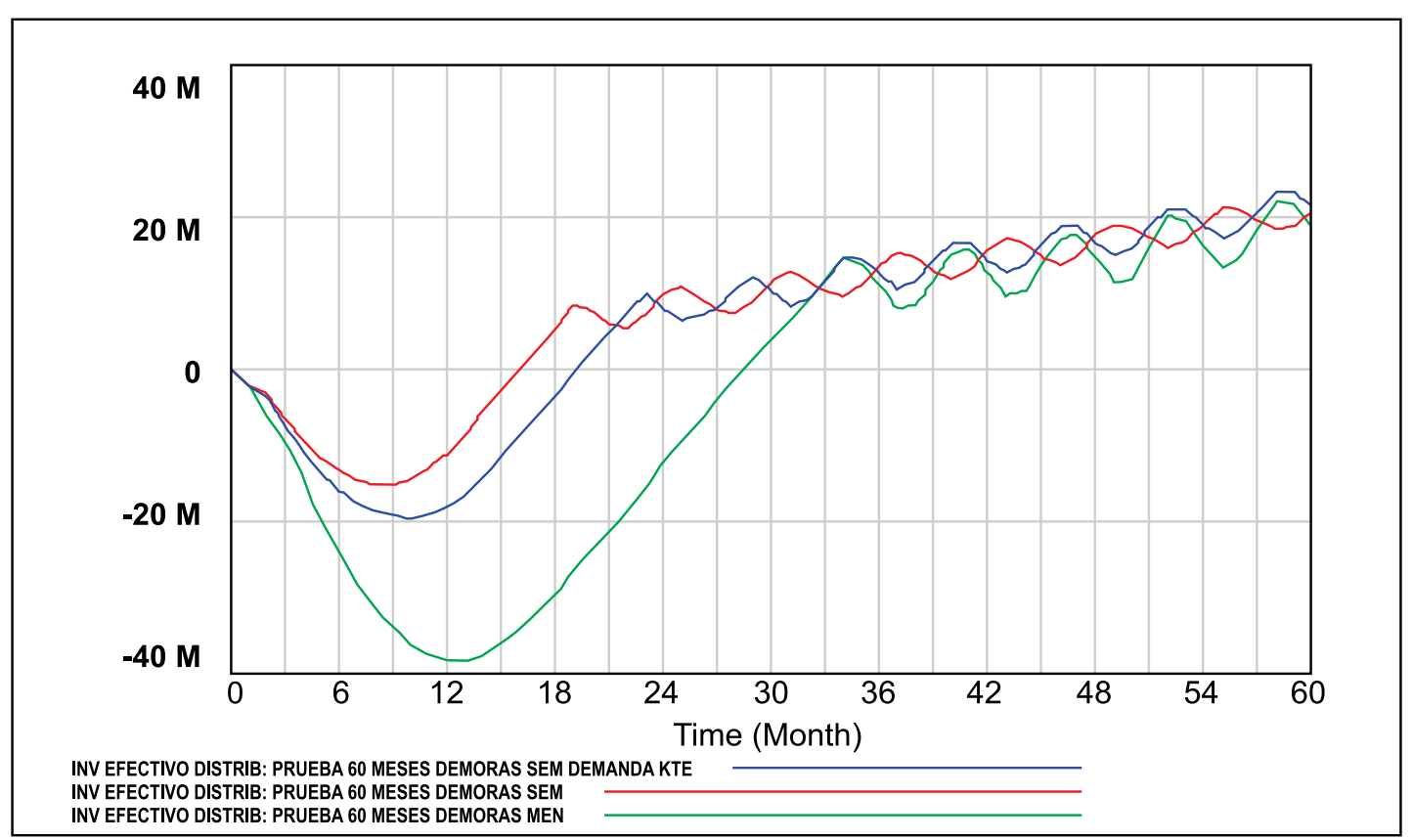

Figura $\mathrm{N}^{\circ}$ 6: Inventario efectivo de distribuidores.

muestra un patrón cíclico a partir del mes 19, con demoras semanales (y a partir del mes 34, con demoras mensuales). El tipo de demanda (constante o estocástica), no implica diferencias significativas en el comportamiento del inventario efectivo, solamente un retraso más.

Se encontró que en el caso de demoras mensuales, presenta una amplificación del "efecto látigo" llegando a un punto más bajo el inventario efectivo, respecto al caso de demoras semanales. En los eslabones distribuidores y fábrica, se presenta un comportamiento oscilatorio a partir del período 19; sin embargo ya no alrededor de un inventario efectivo de 0 , sino alrededor de una línea de tendencia positiva (que ocurre porque la orden de los distribuidores está relacionada de forma linealmente

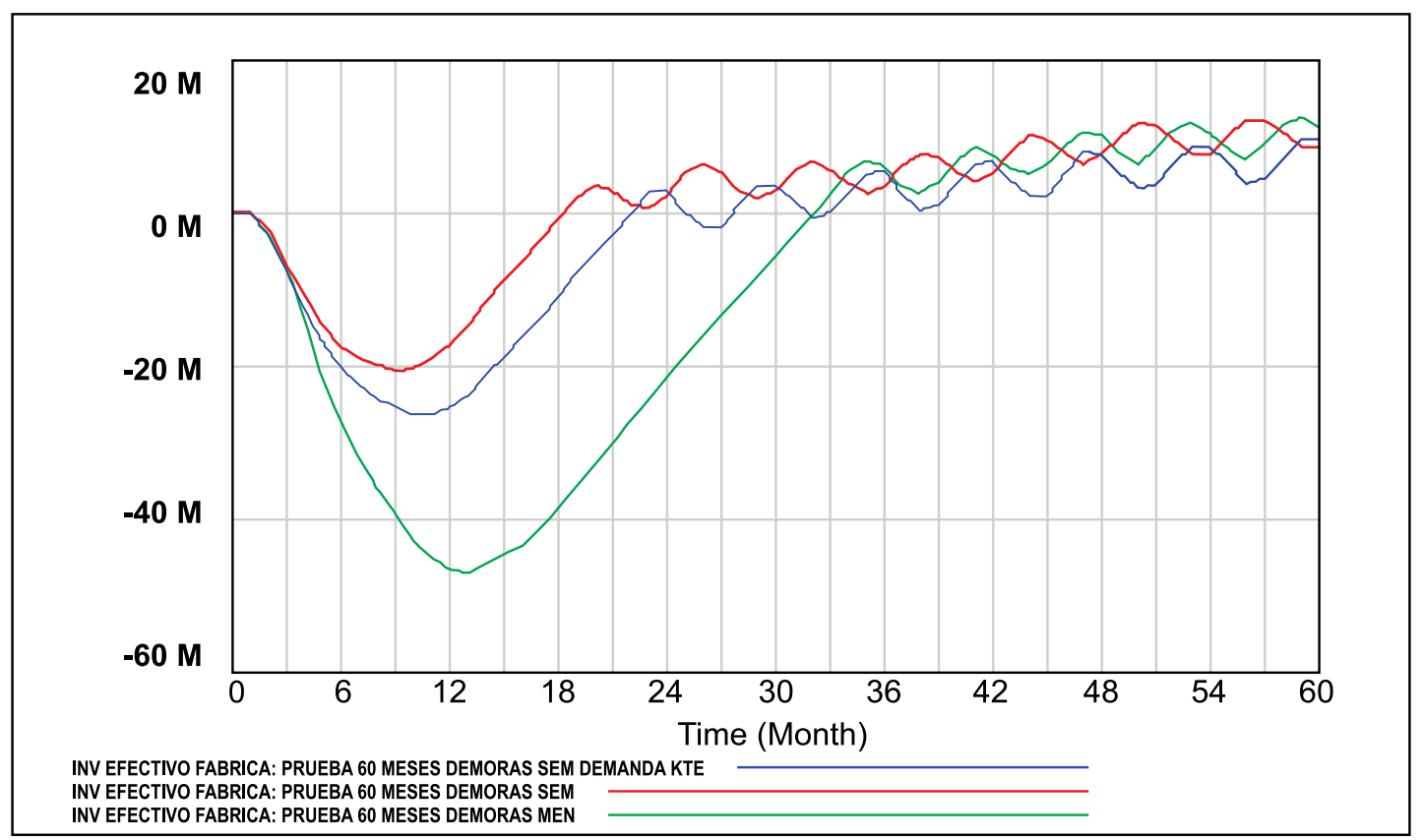

Figura $\mathrm{N}^{\circ} 7$ : Inventario efectivo de fábrica. 
positiva con la orden de los expendios).

Ante un escenario de demanda conocida por todos los eslabones, el eslabón expendios es aquel que se ve relativamente más afectado debido a que el faltante que se presenta en un principio le provoca un faltante que permanece oscilando alrededor del mayor valor que alcanzó el faltante en el primer modelo realizado y que ya no es pertinente cumplir en períodos posteriores. Este valor tiene una relación cercana a 1,9 veces la demanda. El eslabón distribuidores tendría un faltante cercano a los dos millones de cajas en cada uno de los períodos simulados y el eslabón fábrica podría reaccionar en el mismo período que conoce la demanda, así su inventario efectivo sería de 0 unidades.

\section{DISCUSIÓN}

Mediante el desarrollo de este trabajo se determinaron las variables necesarias para modelar el sistema; también se lograron determinar las relaciones causales existentes entre las variables identificadas, mediante la elaboración de un diagrama causal que muestra la dirección y el sentido de cada relación de incidencia (positiva o negativa). Asimismo, se argumentaron los motivos de dichas relaciones y se elaboró un modelo con un componente grafico muy claro (en términos de niveles, flujos y variables auxiliares), según los diagramas de dinámica de sistemas propuestos por Jay Forrester. Este modelo incluye diversas ecuaciones que representan el funcionamiento del sistema por componentes e integra las relaciones entre eslabones durante la distribución de bebidas.

A partir del estudio del sistema y del modelado generado se realizaron varias pruebas sobre escenarios posibles, tales como modificar los tiempos de entrega de producto, asumir otro comportamiento en la demanda y permitir que todos los eslabones conozcan las señales de demanda directamente del mercado tan pronto como se generan. Estas pruebas simularon cómo se comportarían los niveles de inventario efectivo (inventario positivo faltante) en un horizonte de 60 meses, es decir, 5 años.

Por lo anterior, proponemos implementar un conjunto de medidas que detallamos a continuación: i) propiciar la Integración de eslabones -puesto que en la actualidad cada ente actúa motivado por su bienestar particular-, que se lograría mediante la implementación de un sistema de información que envíe la información desde los expendios hasta los responsables de realizar la planeación de la producción; ii) tratar de reducir los tiempos de entrega del producto, lo cual podría lograrse introduciendo mejoras a los procesos productivos y a los procesos administrativos de despacho; iii) utilizar el stock de seguridad para ayudar a cubrir la demanda, porque en la actualidad se presentan períodos con faltante de producto y simultáneamente con inventario de seguridad, cuando deberían ser variables excluyentes.

\section{Agradecimientos}

El agradecimiento especial a la Universidad Continental de Perú por otorgar los recursos necesarios para el desarrollo de esta investigación; también a las empresas Ajeper y Bavaria por suministrar datos imprescindibles para el modelado.

\section{REFERENCIAS BIBLIOGRÁFICAS}

1. Holweg $M$, Disney $S M$, Holmström J, Småros J. Supply chain collaboration: making sense of the strategy continuum, European Management Journal; 2005.

2. Martín-Andino Ramón. El efecto látigo (bullwhip) en las cadenas de suministro y la dependencia de los agentes que las integran. [Tesis doctoral]. Madrid: Universidad Pontificia Comillas; 2006.

3. Sancar Urun. Quantification of the Bullwhip Effect. Department of 
Management Information Systems Bogazici University; 2003.

4. Companys R. Diseño de sistemas productivos y logísticos. EPSEB-UPC; 2005.

5. Stadtler H. Supply Chain Management and Advanced Planning: Concepts, Models, Software, and Case Studies. Springer-Verlag-Berlin; 2008.

6. Forrester JW. Industrial Dynamics, Portland (OR): Productivity Press; 1961.

7. Metters R. Quantifying the bullwhip effect in supply chains. Journal of Operations Management. 1997; 15(2): 89-100

8. Flood R, Jackson M. Creative Problem Solving. Total Systems Intervention. 1 ed. California: John Wiley \& Sons; 1991.

9. Alonso I, Álvarez Y. El proceso de toma de decisiones en entornos complejos: Una aplicación metodológica. España: Universidad de Oviedo, Facultad de Ciencias Económicas; 2000.

10. Torres M, Uribe P. El juego de la cerveza. Chile: Universidad Tecnológica Metropolitana; 2006. 